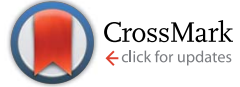

Cite this: RSC Adv., 2016, 6, 99053

Received 16th August 2016

Accepted 13th October 2016

DOI: $10.1039 / c 6 r a 20623 a$

www.rsc.org/advances

\section{Probing thermal expansion coefficients of monolayers using surface enhanced Raman scattering}

\author{
Duan Zhang, $\dagger^{\mathrm{a}}$ Ye-Cun Wu,,$^{\mathrm{a}}$ Mei Yang, ${ }^{\mathrm{a}}$ Xiao Liu, ${ }^{a}$ Cormac Ó Coileáin, ${ }^{\text {abc }}$ \\ Hongjun Xu, ${ }^{\text {ab }}$ Mourad Abid, ${ }^{b}$ Mohamed Abid, ${ }^{b}$ Jing-Jing Wang, ${ }^{c}$ Igor $V$. Shvets, ${ }^{c}$ \\ Haonan Liu, ${ }^{a}$ Zhi Wang, ${ }^{a}$ Hongxing Yin, ${ }^{a}$ Huajun Liu, ${ }^{d}$ Byong Sun Chun, ${ }^{e}$ \\ Xiangdong Zhang ${ }^{a}$ and Han-Chun Wu*a
}

\begin{abstract}
Monolayer transition metal dichalcogenides exhibit remarkable electronic and optical properties, making them candidates for application within flexible nano-optoelectronics, however direct experimental determination of their thermal expansion coefficients (TECS) is difficult. Here, we propose a non-destructive method to probe the TECs of monolayer materials using surface-enhanced Raman spectroscopy (SERS). A strongly coupled Ag nanoparticle over-layer is used to controllably introduce temperature dependent strain in monolayers. Changes in the first-order temperature coefficient of the Raman shift, produced by TEC mismatch, can be used to estimate relative expansion coefficient of the monolayer. As a demonstration, the linear TEC of monolayer $W_{2}$ is probed and is found to be $10.3 \times 10^{-6} \mathrm{~K}^{-1}$, which would appear support theoretical predictions of a small TEC. This method opens a route to probe and control the TECs of monolayer materials.
\end{abstract}

Two dimensional (2D) materials, such as transition metal dichalcogenides (TMDs), have attracted much attention due to their outstanding electronic and optical attributes. ${ }^{\mathbf{1 - 1 0}}$ For integration with existing semiconductor technology 2D TMDs have a natural advantage over graphene, in that they typically possess an energy bandgap, and yet can display high carrier mobilities. The bandgaps of TMDs are thickness dependent, typically displaying a transition from an indirect to directbandgap when the thickness is reduced to a monolayer. ${ }^{2,3,11,12}$ However, a key physical consideration for the application of 2D materials is their thermal expansion coefficient (TEC), which relates changes in dimension to temperature. While many of the optical and electronic properties of TMDs have been well characterized, the thermal properties of many 2D materials remain less explored due to the difficulties associated with experimental measurements. Most materials exhibit positive thermal expansion, expanding when heated and contracting when cooled. However some materials do exhibit negative thermal expansion, and an interesting few exhibit very low

\footnotetext{
${ }^{a}$ Beijing Key Lab of Nanophotonics and Ultrafine Optoelectronic Systems, School of Physics, Beijing Institute of Technology,Beijing 100081, P. R. China.E-mail: wuhc@ bit.edu.cn

${ }^{b}$ KSU-Aramco Center, King Saud University, Riyadh 11451, Saudi Arabia ${ }^{c}$ CRANN, School of Physics, Trinity College, University of Dublin, Dublin 2, Ireland ${ }^{d}$ Institute of Plasma Physics, Chinese Academy of Sciences, Hefei 230031, P. R. China ${ }^{e}$ Division of Industrial Metrology, Korea Research Institute of Standards and Science, Daejeon 305-340, South Korea

$\uparrow$ These authors contributed equally to this work.
}

(less than $2 \times 10^{-6} \mathrm{~K}^{-1}$ ) or zero thermal expansion within specific temperature ranges. ${ }^{13} \mathrm{~A}$ small TEC is highly desirable for applications where there is little tolerance for dimensional change or for systems that experience rapid temperature variations but require consistency, such as for nano-electromechanical devices ${ }^{\mathbf{1 4}}$ or nanosensors. ${ }^{15}$ It is well known that the origin of thermal expansion is anharmonic atomic lattice interactions, where the average interatomic distances increase as higher vibrational energy levels become available and are occupied. Therefore, crystal structure can greatly affect the TEC, for example, diamond is a positive TEC material, ${ }^{16}$ graphite exhibits negative in-plane but positive out-of-plane TECs, ${ }^{17}$ and from experiment and theoretical predictions, graphene is recognized as having a negative TEC over a wide range of temperatures. ${ }^{18-23}$ Other 2D materials such as monolayer hexagonal boron nitride are also predicted to exhibit a negative TEC. ${ }^{21,22}$ On the other hand, 2D TMDs are generally believed to demonstrate positive TECs. ${ }^{23,24}$ Specifically, recent first principles calculations have indicated that the linear TEC of monolayer $\mathrm{WS}_{2}$ is very small. ${ }^{24}$ Bulk $\mathrm{WS}_{2}$ has an indirect bandgap of $1.3 \mathrm{eV}$, whereas a monolayer has direct bandgap of $2.1 \mathrm{eV},{ }^{12}$ and another notable feature is intense photoluminescence (PL) found for monolayer $\mathrm{WS}_{2}{ }^{25}$ These properties suggest that such monolayers have potential for applications within flexible $2 \mathrm{D}$ nano-optoelectronics. However, the claim of a small TEC is yet to be experimentally confirmed due to the difficulties associated with measuring expansion at the length scales associated with 2D materials. For monolayer materials the thermal expansion 
coefficient can be ideally experimentally determined through delicate nanoelectromechanical resonance measurements or direct imaging of rippling of suspended monolayers. ${ }^{26,27}$ However these methods require intricate device fabrication or extensive careful imaging.

Here we propose a relatively simple non-destructive method to probe the TECs of monolayer materials using surfaceenhanced Raman spectroscopy (SERS). A strongly coupled metallic nanoparticle (NP) over-layer is used to controllably introduce strain in the monolayers. The vibrational modes of the monolayer are sensitive to strain resulting from TEC mismatch, which are probed in temperature dependent Raman measurements. For monolayer $\mathrm{WS}_{2}$, the out-of-plane linear TEC is found to be at most $\sim 10.3 \times 10^{-6} \mathrm{~K}^{-1}$, which would appear support theoretical predictions of a small TEC. We also use monolayer $\mathrm{MoS}_{2}$ to show how the magnitude of the strain corresponds to the degree of TEC mismatch. This experimental study suggests how the thermal properties of monolayer materials could be tuned and how the TECs of monolayer materials can be investigated in a nondestructive manner. The method proposed here is robust, and would be suitable for probing the TECs both for supported and freestanding monolayers.

\section{Results and discussion}

Fig. 1a shows a schematic of the proposed method. A strongly coupled metallic nanoparticle (NP) over-layer with a comparatively larger TEC is added to introduce strain in the TMD monolayer. Cooling induces contraction of metal NPs and strains the TMD monolayer. The magnitude of this strain is in proportion to the mismatch between the TECs. Thus the vibrational modes of the monolayer with NPs will be shifted with respect to the bare monolayer, which can be clearly observed during temperature dependent Raman measurements. ${ }^{28,29}$ Since the origin of thermal expansion is anharmonic atomic lattice interactions, a quasi-harmonic approximation can be used to express the change in the phonon frequency $(\omega)$ with temperature $(T)$ in terms of the contribution made by thermal expansion in the lattice. ${ }^{30,31}$

$$
\frac{\partial \omega}{\partial T}=2 a_{0} \alpha \frac{\partial \omega}{\partial a}
$$

where $a_{0}$ is the equilibrium lattice constant, $\alpha$ is the linear thermal expansion coefficient and $\partial \omega / \partial a$ is the variation of phonon frequency with lattice constant $a$. The presence of the NP over-layer should not affect the value of $\partial \omega / \partial a$, which describes the third-order coupling between phonons and longwavelength acoustic phonons for the monolayer, particularly as metals such as Ag are widely used for SERS without notable deleterious aberrations in the Raman spectra. This implies that the dominant contribution to changes in phonon frequency is from the thermal expansion, and it can be inferred that the change is correlated to a change in $\alpha$, i.e. $\partial \omega / \partial T \propto \alpha$. Thus, the change in the phonon frequency relates the TEC of the monolayer to the TEC of the metal, and by comparing the temperature dependent Raman spectra of TMD monolayers with and without metallic NPs we can estimate the TECs of the bare TMD monolayers. We would like to note that in ref. 30, Late et al. suggested the possibility of measuring the TEC by temperature dependent Raman spectra. In their method, $\left(\frac{\partial \omega}{\partial T}\right)$ can be determined from the Raman measurements, while the change with lattice constant $\left(\frac{\partial \omega}{\partial a}\right)$ has to be effectively determined through first-principles density functional theory calculations. ${ }^{30}$ In our proposed method we measure the change in the Raman frequency with temperature with $\left(\chi_{\mathrm{ML}+\mathrm{NP}}\right)$ and without $\left(\chi_{\mathrm{ML}}\right)$ metal nanoparticles. Since the metallic nanoparticles are deposited by an e-beam evaporator and strongly coupled with the monolayer underneath, the thermal expansion for coupled system is mainly controlled by NPs. Thus with nanoparticles eqn (1) becomes

$$
\frac{\partial \omega_{\mathrm{ML}+\mathrm{NP}}}{\partial T}=2 a_{0} \alpha_{\mathrm{NP}} \frac{\partial \omega}{\partial a}
$$

where $\omega_{\text {ML+NP }}$ is the Raman frequency of the combined system and $\alpha_{\mathrm{NP}}$ is the expansion coefficient of the metallic NPs. Since $\left(\frac{\partial \omega}{\partial a}\right)$ should not change for SERS measurements, we can calculate the expansion coefficient of monolayer from the relative change

$$
\alpha_{\mathrm{ML}} \simeq \frac{\chi_{\mathrm{ML}}}{\chi_{\mathrm{ML}+\mathrm{NP}}} \alpha_{\mathrm{NP}}
$$

As the thermal expansion coefficient of the metal is known, it allows us to estimate the TECs of the bare monolayers using SERS. The advantages of our proposed method can be summarized as follows. First, the metallic NPs act as a reference point to determine the TEC of the monolayer. Second, SERS allows us to check for local strain within the monolayer to ensure strong coupling between the nanoparticles and monolayer, and gives a better signal to noise ratio. Third, metal has a high thermal conductivity; it can help prevent burning of the monolayer when laser is switched on. Fourth, the approach we propose could be used for wide variety of monolayers, even layers with mixed compositions, provided there is strong coupling with a nanoparticles over-layer. While it is known that SERS can suffer from issues of reproducibility, in our proposed method the nanoparticles are prepared by e-beam evaporation, thus provided the same growth conditions are maintained the distribution on the surface should be consistent. Furthermore, it is the intensity of the SERS signal that typically suffers from issues of reproducibility, which is not a factor within our calculations. The relative change in the peak position is the factor under consideration, and in fact similar consistent behaviour was observed for more than five different experiments.

To demonstrate the proposed method, the TEC of monolayer $\mathrm{WS}_{2}$ was probed using SERS, stimulated by a coating of Ag NPs on top, which was deposited by e-beam evaporation. The largescale continuous $\mathrm{WS}_{2}$ monolayers were produced by sulfurization of monolayer oxide films, further details of sample preparation and characterization can be found in the methods 

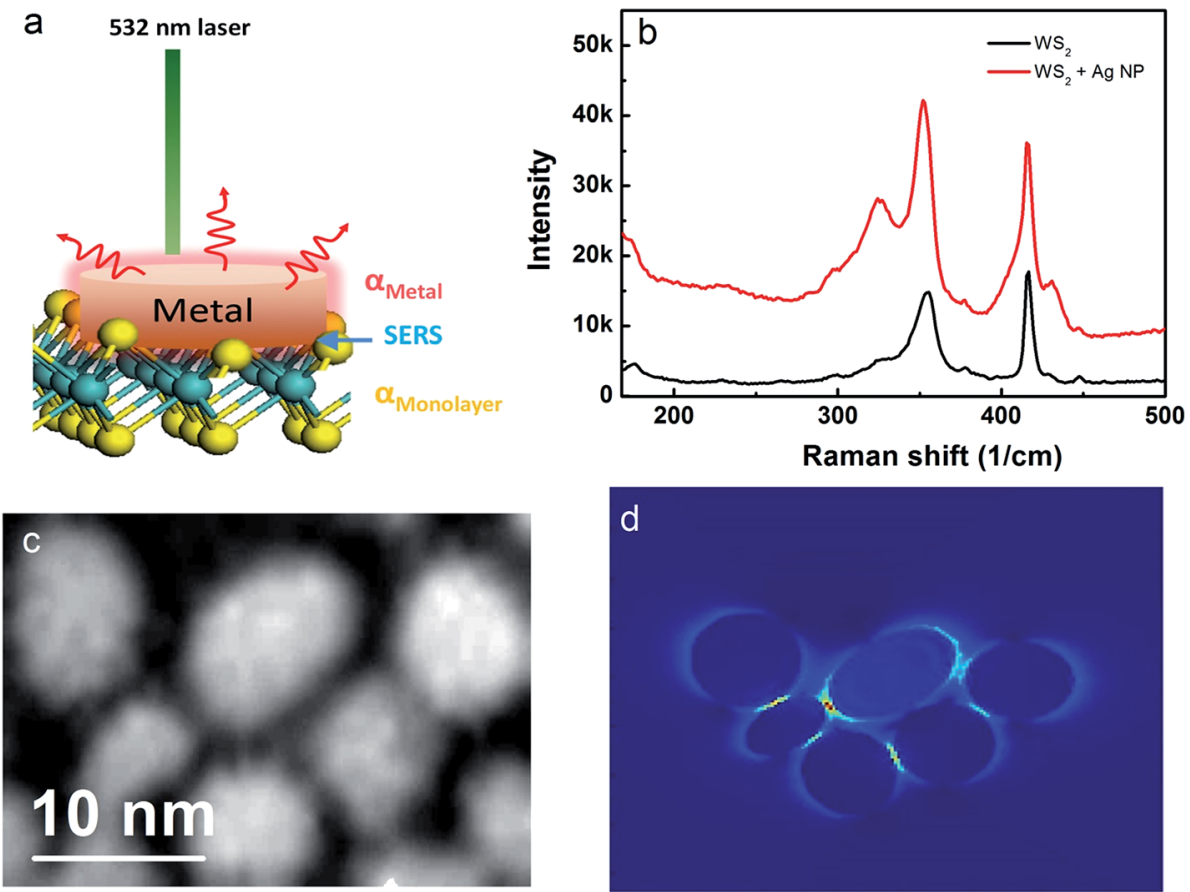

Fig. 1 SERS of monolayer $W_{2}$ at room temperature. (a) Schematic drawing of the experimental setup of the SERS measurements of WS 2 . (b) Raman spectra of monolayer $W_{2}$ with (Red) and without (Black) $5 \mathrm{~nm}$ nominally thick Ag NPs on top. Arrow indicates the splitting of the $A^{\prime}{ }_{1}(\Gamma)$ mode. (c) SEM image of $5 \mathrm{~nm}$ nominally thick Ag NPs on monolayer $\mathrm{WS}_{2}$. (d) Simulated electric field cartography at the surface of the area marked in Fig. 1c with an excitation wavelength of $532 \mathrm{~nm}$.

section and elsewhere. ${ }^{32}$ Fig. $1 \mathrm{~b}$ displays the room temperature Raman spectra for monolayer $\mathrm{WS}_{2}$ without and with Ag NPs. The Raman spectrum displays peaks for the in-plane active modes $\mathrm{E}^{\prime}(T)$ and out-of-plane modes $\mathrm{A}_{1}^{\prime}(\Gamma)$ at around $356 \mathrm{~cm}^{-1}$ and $417 \mathrm{~cm}^{-1}$, respectively, and the second-order zone-edge phonon peak $2 \mathrm{LA}(\mathrm{M})$ at approximately $353 \mathrm{~cm}^{-1}$. Compared with bulk $\mathrm{WS}_{2}{ }^{33}$ the out-of-plane mode $\mathrm{A}^{\prime}{ }_{1}(\Gamma)$ is red-shifted from 421 to $417 \mathrm{~cm}^{-1}$, resulting from the absence of van der Waals interactions between the layers. As would be expected, the addition of the $5 \mathrm{~nm}$ thick Ag NP layer leads to a clear enhancement of Raman intensity, as shown in Fig. 1b. However, this enhancement is also accompanied by a slight red-shift of the $\mathrm{E}^{\prime}(T)$ mode and additional peaks appear, caused by a strain of approximately $1 \%$ in the $\mathrm{WS}_{2}$ induced by the NPs. ${ }^{32,34}$ The modification of the Raman spectra by the Ag NP over-layer can be can be understood considering two contributing factors; strain and electric field enhancement. First considering strain, the application strain can frustrate the lattice symmetry, leading to peak splitting in the Raman spectra. The application of tensile strain will predominantly lead to changes in the inplane vibrational modes, while mechanical or compressive strain, such as that exerted by the Ag NPs on top, will lead to greater modification of the out of plane modes. The addition of the Ag results in several sources of stress in the monolayers. The $\mathrm{Ag}$ nanoparticles grow in an island formation, as seen in Fig. 1c, were atom clusters nucleate on the monolayer surface and grow forming isolated islands as long as there is exposed surface, and since $\mathrm{Ag}$ is quite mobile even near room temperature, larger islands will grow at the expense of the shrinking of small islands. $^{35}$ The thermodynamic processes, such as surface tension, that drive the formation of the NPs will introduce stress between the Ag and monolayer. The area directly under the NPs will be the most highly strained, with a gradient at the edges towards uncovered areas. However, interestingly, it has been experimentally observed that the degree mechanical strain is largely independent of the NP size. ${ }^{34}$ Notably strain from lattice mismatch is not expected to be a significant contributing factor to within Ag NP films grown by e-beam. ${ }^{36}$ However, during temperature dependent measurements strain can also be generated due to the mismatch in the TECs of the materials. For these experiments, the high thermal conductivity of Ag means that a thermal gradient is not expected, and since the bonding of $\mathrm{WS}_{2}$ to the $\mathrm{Al}_{2} \mathrm{O}_{3}$ substrate is quite weak it is expected that strain from the Ag NPs will be the dominant factor. ${ }^{37}$

Considering the electric field enhancement, in the vicinity of the $\mathrm{Ag}$ NPs the electric field will be enhanced due to the collective oscillations of the conduction electrons. Thus the scattering process is enhanced by a factor of $\left(E_{\text {local }} / E_{0}\right)^{4}$, where $E_{0}$ is the strength of the incident $E$-field and $E_{\text {local }}$ is the strength of the total local electric field in the presence of the metallic $\mathrm{NPs}^{34,38,39}$ For example, the intensity of the 2LA(M) mode is enhanced by a factor of 3 . Using finite-difference time-domain (FDTD) methods, we simulated the electrical field distribution of an area of monolayer $\mathrm{WS}_{2}$ containing several $5 \mathrm{~nm}$ nominal thickness Ag NPs (Fig. 1d). It is clear from the figure that the strength of the localized electric field depends on the size, shape and interactions between the individual NPs. The lightning rod effect is an important factor in determining the 
strength of the electric field, which causes the field to be dramatically enhanced around sharp edges.

To assess the influence temperature has on the expansion of $\mathrm{WS}_{2}$ we focus on two Raman peaks, the in-plane $\mathrm{E}^{\prime}(\Gamma)$ and the out-of-plane $\mathrm{A}_{1}^{\prime}(\Gamma)$ modes. The Raman spectra of both the $\mathrm{E}^{\prime}(\Gamma)$ and $\mathrm{A}^{\prime}{ }_{1}(\Gamma)$ modes blue shift to higher wave-numbers when the temperature is decreased from $300 \mathrm{~K}$ and $120 \mathrm{~K}$, as seen in respectively in Fig. 2a and b for $\mathrm{WS}_{2}$ with $\mathrm{Ag}$ NPs. This trend can be further illustrated by plotting the peak position of the Raman shifts against temperature as shown in Fig. $2 \mathrm{c}$ and d. It is worth noting that the 2LA(M) mode (Fig. 2c) also follows a similar dependence and that similar blue shifts were observed for $\mathrm{WS}_{2}$ without Ag (Fig. 3a). The shift of the Raman peaks with temperature is mainly due to thermal expansion and the temperature contribution from anharmonicity. To quantify this behavior, the temperature dependent Raman shift for the $\mathrm{E}^{\prime}(\Gamma)$ and $\mathrm{A}^{\prime}{ }_{1}(T)$ modes can be fitted using the Grüneisen model: ${ }^{40}$

$$
\omega(T)=\omega_{0}+\chi T
$$

where $\omega_{0}$ is the peak position at $0 \mathrm{~K}$ and $\chi$ is the first-order temperature coefficient (i.e. $\partial \omega / \partial T$ ). Thus from linear fittings of the plots, the first order temperature coefficients for the $\mathrm{E}^{\prime}(\Gamma)$ and $\mathrm{A}_{1}^{\prime}(\Gamma)$ modes of monolayer $\mathrm{WS}_{2}$ on sapphire without Ag NPs are approximately -0.009 and $-0.006 \mathrm{~cm}^{-1} \mathrm{~K}^{-1}$, respectively, which are consistent with previously reported values. ${ }^{\mathbf{4 1 - 4 3}}$ With
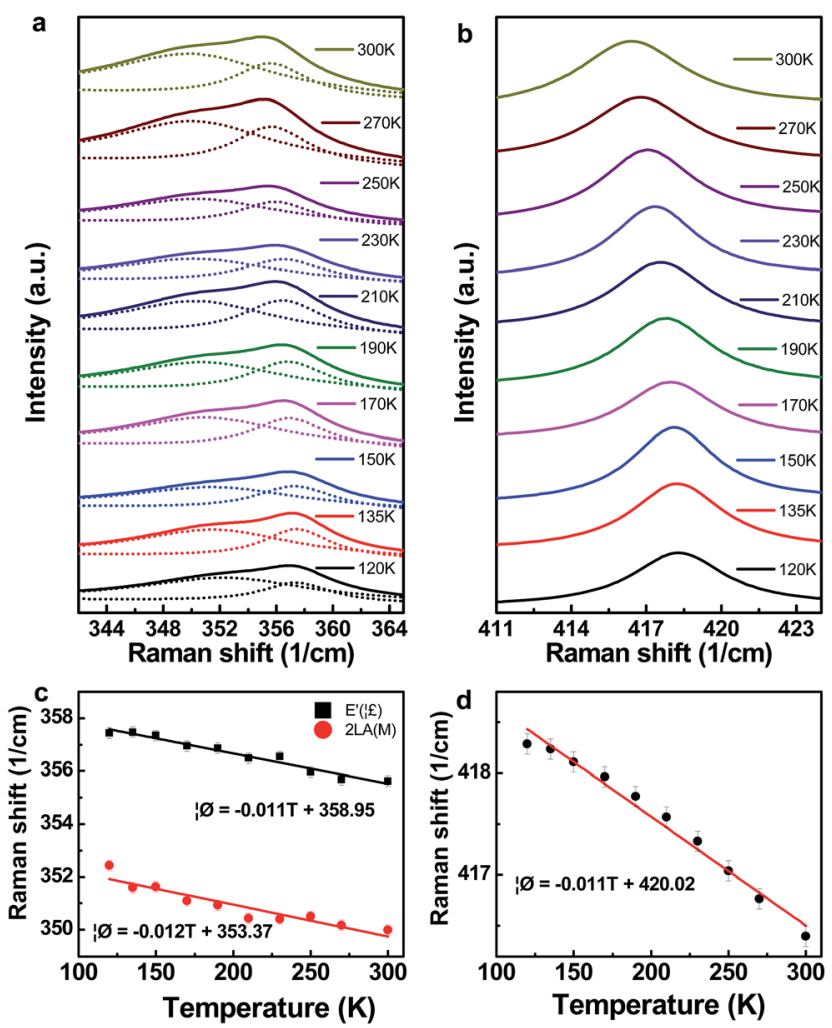

Fig. 2 Temperature dependent Raman shifts of monolayer $\mathrm{WS}_{2}$ with 5 $\mathrm{nm}$ nominally thick Ag NPs on top. Raman spectra for (a) $\mathrm{E}^{\prime}(T)$ mode, and (b) $\mathrm{A}_{1}^{\prime}(\Gamma)$ mode recorded at various temperatures from $120 \mathrm{~K}$ to $300 \mathrm{~K}$. Variation of Raman frequencies as a function of temperature for (c) $\mathrm{E}^{\prime}(\Gamma), 2 \mathrm{LA}(\mathrm{M})$ and $(\mathrm{d}) \mathrm{A}_{1}^{\prime}(\Gamma)$ modes.
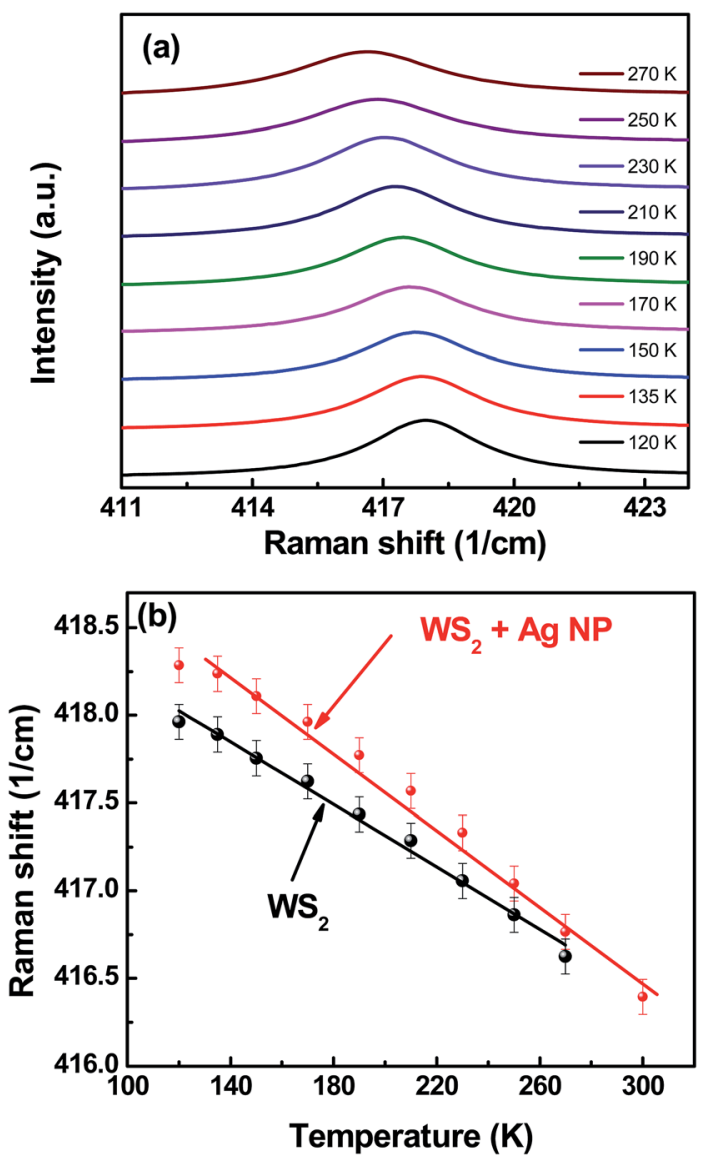

Fig. 3 Temperature dependent Raman shifts of bare monolayer $\mathrm{WS}_{2}$. (a) Raman spectra of $\mathrm{A}_{1}^{\prime}(\Gamma)$ mode recorded for various temperatures ranging from $110 \mathrm{~K}$ to $300 \mathrm{~K}$. (b) Variation of Raman frequencies as a function of temperature for the $\mathrm{A}_{1}^{\prime}(\Gamma)$ mode for $\mathrm{WS}_{2}$ and $\mathrm{WS}_{2}+\mathrm{Ag}$ NP.

the addition $5 \mathrm{~nm}$ of $\mathrm{Ag}$ NPs on top the first-order temperature coefficients for the $\mathrm{E}^{\prime}(\Gamma)$ and $\mathrm{A}_{1}^{\prime}(\Gamma)$ modes are significantly modified, becoming -0.011 and $-0.011 \mathrm{~cm}^{-1} \mathrm{~K}^{-1}$, respectively. Plotting the temperature dependence of the $\mathrm{A}^{\prime}{ }_{1}(\Gamma)$ mode with and without Ag NPs clearly reveals the increasing difference with decreasing temperature (Fig. 3b).

Considering the quasi-harmonic approximation discussed previously, ${ }^{30,31}$ from eqn (1) we know $\chi \propto \alpha$. It should be noted that as the $\mathrm{WS}_{2}$ layer is only weakly bound to the $\mathrm{Al}_{2} \mathrm{O}_{3}$, the

Table 1 Thermal expansion coefficients and thermal conductivity of $\mathrm{Au}, \mathrm{Ag}, \mathrm{Pt}, \mathrm{MoS}_{2}$, and $\mathrm{WS}_{2}$

\begin{tabular}{lll}
\hline Material & $\begin{array}{l}\alpha_{\mathrm{L}} \\
\left(10^{-6} \mathrm{~K}^{-1}\right)\end{array}$ & $\begin{array}{l}\text { Thermal conductivity } \\
\left(\mathrm{W}(\mathrm{m} \mathrm{K})^{-1}\right)\end{array}$ \\
\hline $\mathrm{Au}$ & $14.2^{a}$ & $314^{d}$ \\
$\mathrm{Ag}$ & $18.9^{a}$ & $406^{d}$ \\
$\mathrm{Pt}$ & $8.8^{a}$ & $71.6^{d}$ \\
$\mathrm{MoS}_{2}$ & $24.4^{b}$ & $34^{e}$ \\
$\mathrm{WS}_{2}$ & $15.96^{c}$ & $32^{f}$
\end{tabular}

${ }^{a}$ Ref. $45 .{ }^{b}$ Ref. $24 .{ }^{c}$ Ref. $24{ }^{d}$ Ref. $51 .{ }^{e}$ Ref. $48{ }^{f}$ Ref. 41. 
monolayer should be initially relaxed and not strained by the substrate for both samples. ${ }^{35}$ The volumetric thermal expansion coefficients of bulk $\mathrm{Ag}$ and the $\mathrm{WS}_{2}$ are relatively well matched $^{\mathbf{2 4 , 4 4 , 4 5}}$ (see Table 1), however the change in $\chi$ for the $\mathrm{E}^{\prime}(\Gamma)$ and $\mathrm{A}_{1}^{\prime}(\Gamma)$ modes, with the addition of Ag NPs, suggests compressive strain with decreasing temperature. This would occur if the TEC of $\mathrm{Ag}$ was greater than that of the $\mathrm{WS}_{2}$ monolayer, as the $\mathrm{Ag}$ would contract at a faster rate, suggesting that the TEC of monolayer $\mathrm{WS}_{2}$ is significantly less than that of the bulk. For the $\mathrm{A}^{\prime}{ }_{1}(\Gamma)$ mode the blue shift of $0.3 \mathrm{~cm}^{-1}$ corresponds to compressive strain of approximately $3 \%{ }^{46}$ From the $\chi$ values of the $\mathrm{A}_{1}^{\prime}(\Gamma)$ mode with $\left(\chi_{\mathrm{Ag}}\right)$ and without $\left(\chi_{\mathrm{WS}_{2}}\right) \mathrm{Ag}$, using eqn (3) we can calculate that the out-of-plane $\alpha$ for monolayer $\mathrm{WS}_{2}$ is at most 55\% the TEC of Ag, which corresponds to a TEC of 10.3
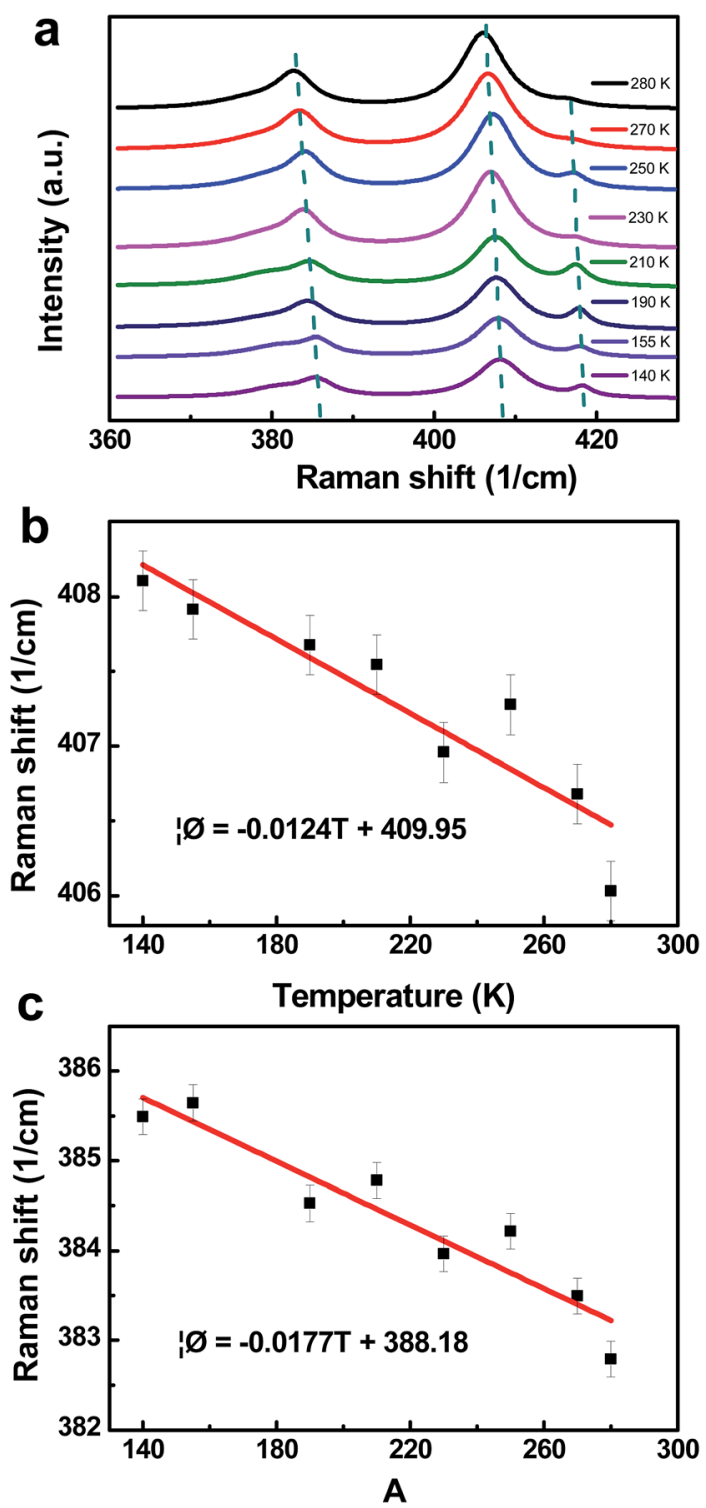

Fig. 4 Temperature dependent Raman shifts of monolayer $\mathrm{MoS}_{2}$ with $5 \mathrm{~nm}$ nominally thick Ag NPs on top. (a) Raman spectra recorded at various temperatures from $120 \mathrm{~K}$ to $300 \mathrm{~K}$. (b) and (c) are variation of Raman frequencies as a function of temperature for $\mathrm{E}^{\prime}(\Gamma)$ and $\mathrm{A}_{1}^{\prime}(\Gamma)$ modes respectively. $\times 10^{-6} \mathrm{~K}^{-1}$. Our experimental results appear to support recent first principles calculations. ${ }^{24}$

To demonstrate the versatility of this approach, an analogous experiment was also performed with monolayer $\mathrm{MoS}_{2}$, where the thermal expansion coefficient of $\operatorname{MoS}_{2}\left(\alpha_{\mathrm{Mos}_{2}}\right)$ is slightly larger than that of $\mathrm{Ag}\left(\alpha_{\mathrm{Ag}}\right)$ (Table 1). Fig. 4a shows the temperature dependent Raman spectra for a monolayer of $\mathrm{MoS}_{2}$ with $5 \mathrm{~nm}$ of Ag NPs grown on top. Fig. $4 \mathrm{~b}$ and c plot peak position of the temperature dependent Raman shifts for the $\mathrm{E}^{\prime}(\Gamma)$ and $\mathrm{A}_{1}^{\prime}(\Gamma)$ modes with Ag NPs. As $\alpha_{\mathrm{Mos}_{2}}>\alpha_{\mathrm{Ag}}$, dramatic modification of $\chi$ is not expected. From Fig. $4 \mathrm{~b}$ and $\mathrm{c}$, the $\chi$ values for the $\mathrm{E}^{\prime}(\Gamma)$ and $\mathrm{A}^{\prime}{ }_{1}(\Gamma)$ modes are $\sim-0.012$ and $\sim-0.018 \mathrm{~cm}^{-1} \mathrm{~K}^{-1}$ with $\mathrm{Ag}$, which are consistent with reports for $\mathrm{MoS}_{2}$ with Ag. ${ }^{4-50}$ The lack of a significant change in $\chi$ with the addition of the NPs suggests the $\mathrm{MoS}_{2}$ is less strained at low temperature and thus TECs of Ag and $\mathrm{MoS}_{2}$ are broadly similar.

\section{Conclusion}

We used temperature dependent SERS to probe the TECs of monolayer TMDs. Temperature dependent strain in the monolayer is induced by the TEC mismatch between the monolayer and the strongly coupled Ag NP over-layer. This allows used to access the linear TECs of the monolayers from changes in the first order linear thermal coefficients of in-plane and out of plane modes. Using this approach we demonstrated the small TEC of monolayer $\mathrm{WS}_{2}$. This method could be expanded and refined for a broad range of NP and monolayer materials, and also suggests how expansion of monolayers could be controlled.

\section{Methods}

The monolayer $\mathrm{WO}_{3}$ was deposited on single crystal $\alpha-\mathrm{Al}_{2} \mathrm{O}_{3}$ (0001) substrates at $400{ }^{\circ} \mathrm{C}$ using an e-beam heated $\mathrm{WO}_{3}$ source in a MBE system (DCA) with in an oxygen partial pressure of $5 \times$ $10^{-6}$ Torr. Reflection high electron diffraction (RHEED) was employed to monitor and establish growth mode. After growth, the $\mathrm{WO}_{3}$ monolayers were annealed at $650{ }^{\circ} \mathrm{C}$, with the same pressure for 30 minutes. The oxide samples were removed from vacuum and sulfurized in a furnace using $10 \% \mathrm{H}_{2}$ in argon gas as the carrier. $\mathrm{MoS}_{2}$ was grown using a similar procedure. The Ag nanoparticles with a $5 \mathrm{~nm}$ nominal thickness were deposited on the monolayers at room temperature by e-beam evaporation. The high quality and chemical composition of the samples was verified before and after sulfurization by X-ray photoemission spectroscopy (XPS). Raman spectroscopy, and PL spectroscopy were used to further evaluate the thickness of the $\mathrm{WS}_{2}$ layer. Raman measurements were performed using a Renishaw RM1000 spectrometer with an excitation wavelength of $532 \mathrm{~nm}$. The temperature of sample was controlled by a Linkam LTHMS600E microscopy stage which has a temperature range of $-196{ }^{\circ} \mathrm{C}$ to $600{ }^{\circ} \mathrm{C}$. During the measurement, we used $50 \times$ Leica long-focus objective lens and the power of laser was set to be $1 \%$. A bandgap of $\sim 2 \mathrm{eV}$ is observed indicating the grown $\mathrm{WS}_{2}$ is monolayer in nature. ${ }^{25}$ 


\section{Author contributions}

H. C. W. supervised the project and led the overall effort. D. Z., Y. C. W., M. Y. and X. L. carried out the Raman measurements. C. O., MOH. A., and I. V. S. grew the sample. MOU. A. performed the FDTD simulation. H. L. and Z. W. deposited the Ag nanoparticles. H. X. Y. carried out the SEM characterization. D. Z., Y. C. W., J. J. W., H. J. X., and H. C. W. analyzed the data. H. J. L. and B. S. C. commented the paper. X. D. Z. gave scientific advice. H. C. W. wrote the paper. All authors discussed the results and commented on the manuscript.

\section{Competing financial interests}

The authors declare there is no competing financial interest.

\section{Acknowledgements}

This work was supported by the Beijing Institute of Technology Research Fund Program for Young Scholars and National Plan for Science and technology (No. NPST 2472-02 and NPST 252902) of King Abdulaziz City for Science and Technology. H. C. W., $\mathrm{MOH}$. A., and MOU. A. thank Saudi Aramco for the financial support (project No. 6600028398).

\section{References}

1 K. Novoselov, D. Jiang, F. Schedin, T. Booth, V. Khotkevich, S. Morozov and A. Geim, Proc. Natl. Acad. Sci. U. S. A., 2005, 102, 10451.

2 Q. Wang, K. Kalantar-Zadeh, A. Kis, J. Coleman and M. Strano, Nat. Nanotechnol., 2012, 7, 699-712.

3 K. Mak, C. Lee, J. Hone, J. Shan and T. Heinz, Phys. Rev. Lett., 2010, 105, 136805.

4 G. Eda and S. Maier, ACS Nano, 2013, 7, 5660.

5 B. Radisavljevic, A. Radenovic, J. Brivio, V. Giacometti and A. Kis, Nat. Nanotechnol., 2011, 6, 147.

6 D. Xiao, G. Liu, W. Feng, X. Xu and W. Yao, Phys. Rev. Lett., 2012, 108, 196802.

7 K. Mak, K. McGill, J. Park and P. McEuen, Science, 2014, 344, 1489.

8 R. Mas-Ballesté, C. Gómez-Navarro, J. Gómez-Herrero and F. Zamora, Nanoscale, 2011, 3, 20.

9 A. Rodin, A. Carvalho and A. Castro Neto, Phys. Rev. Lett., 2014, 112, 176801.

10 W. Zhang, Q. Wang, Y. Chen, Z. Wang and A. Wee, 2D Mater., 2016, 3, 022001.

11 W. Zhao, Z. Ghorannevis, L. Chu, M. Toh, C. Kloc, P. Tan and G. Eda, ACS Nano, 2013, 7, 791.

12 A. Kuc, N. Zibouche and T. Heine, Phys. Rev. B: Condens. Matter Mater. Phys., 2011, 83, 245213.

13 R. Roy, D. Agrawal and H. McKinstry, Annu. Rev. Mater. Sci., 1989, 19, 59.

14 S. Jun, X. Huang, M. Manolidis, C. Zorman, M. Mehregany and J. Hone, Nanotechnology, 2006, 17, 1506.

15 Y. Gao and Y. Bando, Nature, 2002, 415, 599.

16 R. Krishnan, Nature, 1944, 154, 486.
17 D. Tsang, B. Marsden, S. Fok and G. Hall, Carbon, 2005, 43, 2902.

18 V. Singh, S. Sengupta, H. Solanki, R. Dhall, A. Allain, S. Dhara, P. Pant and M. Deshmukh, Nanotechnology, 2010, 21, 165204.

19 D. Yoon, Y. Son and H. Cheong, Nano Lett., 2011, 11, 3227.

20 A. Balandin, Nat. Mater., 2011, 10, 569.

21 K. Zakharchenko, J. Los, M. Katsnelson and A. Fasolino, Phys. Rev. B: Condens. Matter Mater. Phys., 2010, 81, 235439. 22 L. Feng Huang and Z. Zeng, J. Appl. Phys., 2013, 113, 083524.

23 C. Sevik, Phys. Rev. B: Condens. Matter Mater. Phys., 2014, 89, 035422.

24 Y. Ding and B. Xiao, RSC Adv., 2015, 5, 18391.

25 N. Peimyoo, J. Shang, C. Cong, X. Shen, X. Wu, E. Yeow and T. Yu, ACS Nano, 2013, 7, 10985.

26 W. Bao, F. Miao, Z. Chen, H. Zhang, W. Jang, C. Dames and C. Lau, Nat. Nanotechnol., 2009, 4, 562.

27 V. Singh, S. Sengupta, H. Solanki, R. Dhall, A. Allain, S. Dhara, P. Pant and M. Deshmukh, Nanotechnology, 2010, 21, 165204.

28 C. Cong and T. Yu, Nat. Commun., 2014, 5, 4709.

29 I. Calizo, A. Balandin, W. Bao, F. Miao and C. Lau, Nano Lett., 2007, 7, 2645.

30 D. Late, S. Shirodkar, U. Waghmare, V. Dravid and C. Rao, ChemPhysChem, 2014, 15, 1592.

31 M. Upadhyay Kahaly and U. Waghmare, Appl. Phys. Lett., 2007, 91, 023112.

32 D. Zhang, Y. Wu, M. Yang, X. Liu, C. Coileáin, M. Abid, M. Abid, J. Wang, I. Shvets, H. Xu, B. Chun, H. Liu and H. Wu, Sci. Rep., 2016, 6, 30320.

33 X. Zhang, X. Qiao, W. Shi, J. Wu, D. Jiang and P. Tan, Chem. Soc. Rev., 2015, 44, 2757.

34 Y. Sun, K. Liu, X. Hong, M. Chen, J. Kim, S. Shi, J. Wu, A. Zettl and F. Wang, Nano Lett., 2014, 14, 5329.

35 S. Seel, PhD thesis, Massachusetts Institute of Technology, 2002.

36 C. Gong, C. Huang, J. Miller, L. Cheng, Y. Hao, D. Cobden, J. Kim, R. Ruoff, R. Wallace, K. Cho, X. Xu and Y. Chabal, ACS Nano, 2013, 7, 11350.

37 L. Su, Y. Yu, L. Cao and Y. Zhang, Nano Res., 2015, 8, 2686. 38 H. Xu, J. Aizpurua, M. Käll and P. Apell, Phys. Rev. E: Stat. Phys., Plasmas, Fluids, Relat. Interdiscip. Top., 2000, 62, 4318. 39 E. Le Ru and P. Etchegoin, MRS Bull., 2013, 38, 631.

40 E. Zouboulis and M. Grimsditch, Phys. Rev. B: Condens. Matter Mater. Phys., 1991, 43, 12490.

41 M. Thripuranthaka, R. Kashid, C. Sekhar Rout and D. Late, Appl. Phys. Lett., 2014, 104, 081911.

42 T. M and D. Late, ACS Appl. Mater. Interfaces, 2014, 6, 1158. 43 N. Peimyoo, J. Shang, W. Yang, Y. Wang, C. Cong and T. Yu, Nano Res., 2014, 8, 1210.

44 A. Matthäus, J. Electrochem. Soc., 1997, 144, 1013.

45 D. Lide, CRC handbook of chemistry and physics, CRC Press, Boca Raton, FL, 1994.

46 Y. Wang, C. Cong, W. Yang, J. Shang, N. Peimyoo, Y. Chen, J. Kang, J. Wang, W. Huang and T. Yu, Nano Res., 2015, 8, 2562. 
47 N. Lanzillo, A. Glen Birdwell, M. Amani, F. Crowne, P. Shah, S. Najmaei, Z. Liu, P. Ajayan, J. Lou, M. Dubey, S. Nayak and T. O'Regan, Appl. Phys. Lett., 2013, 103, 093102.

48 S. Sahoo, A. Gaur, M. Ahmadi, M. Guinel and R. Katiyar, J. Phys. Chem. C, 2013, 117, 9042.

49 S. Najmaei, P. Ajayan and J. Lou, Nanoscale, 2013, 5, 9758.
50 W. Zhao, Z. Ghorannevis, K. Amara, J. Pang, M. Toh, X. Zhang, C. Kloc, P. Tan and G. Eda, Nanoscale, 2013, 5, 9677.

51 F. Sears, M. Zemansky and H. Young, University Physics, Addison-Wesley Pub. Co., Reading, Mass, 1987. 